Musée des Beaux-Arts de Chartres, 'Uvea-Wallis : Une île pêchée par les dieux

Musée des Beaux-Arts de Chartres. Catalogue de l'exposition présentée en $2000 ; 160$ p., lexique, bibliographie, 154 illustrations, 2 cartes

Marie-Noëlle Ottino-Garanger

\title{
CpenEdition
}

Journals

Édition électronique

URL : http://journals.openedition.org/jso/1634

DOI : $10.4000 /$ jso. 1634

ISSN : 1760-7256

Éditeur

Société des océanistes

Édition imprimée

Date de publication : 1 décembre 2001

Pagination : 227-228

ISSN : 0300-953x

Référence électronique

Marie-Noëlle Ottino-Garanger, « Musée des Beaux-Arts de Chartres, 'Uvea-Wallis : Une île pêchée par les dieux », Journal de la Société des Océanistes [En ligne], 113 | Année 2001-2, mis en ligne le 27 mai 2008, consulté le 24 septembre 2020. URL : http://journals.openedition.org/jso/1634 ; DOI : https://doi.org/ $10.4000 / j s 0.1634$ 
«Un homme ne peut laisser une femme se mettre au-dessus de lui sous peine de voir son ventre se mettre alors à grossir comme dans une grossesse » (p. 315). Cette croyance n'est pas propre aux régions linguistiques dans lesquelles a enquêté Christine Salomon. Nous l'avons entendue un peu partout dans tous les pays kanak. Marcel Mauss dans Les techniques $d u$ corps esquissait déjà des pistes d'investigation sur les techniques de reproduction ${ }^{1}$. Dans la même observation ethnolinguistique, la pollution féminine, par les maladies sexuellement transmissibles, se dit en paicî wâtemû (case féminine) par opposition à la grande case masculine. Les pratiques des femmes kanak démontrent des positions moins figées vis-à-vis des règles traditionnelles de la domination masculine. $\mathrm{La}$ conclusion semble ouverte puisqu'elle finit sur une note positive : « les formes de vie des femmes, à l'heure actuelle, sont moins verrouillées qu'il n'y paraît de prime abord » (p. 336).

On laissera au lecteur la liberté de lecture. Nous retiendrons, à propos de ce livre collectif, l'innovation dans la pratique scientifique. Nous émettrons des réserves et des désaccords sur certaines enquêtes ou sur quelques points. Par exemple, la volonté, de la part de certains jeunes docteurs, d'avoir des partis pris de choix d'école. Ou d'être pris dans un formalisme et une logique scolastique qui consiste à poser d'entrée de jeu que la thèse avancée est nouvelle par rapport à des travaux précédents. Mais, comme disait Jacobson, la science est polémique. L'ouvrage suscite des réflexions et des prolongements dans l'enquête. En ce sens, on retrouve l'infinité de la parole qu'évoquait l'avantpropos d'Octave Togna.

\section{Hamid MoKadDEM}

IFMNC, Nouméa (Nouvelle-Calédonie)

'Uvea-Wallis : Une île pêchée par les dieux. Musée des Beaux-Arts de Chartres. Catalogue de l'exposition présentée en 2000 ; 160 p., lexique, bibliographie, 154 illustrations, 2 cartes.

Pour la première fois une exposition présentait en France ce Territoire français d'Outre-mer. Ce point remarquable sert en quelque sorte d'entrée en matière au catalogue, seul ouvrage à présenter ainsi le panorama actuel de cette île du Pacifique et son ancienne culture. Claude Stéfani, attaché de conservation au musée, et Hélène Guiot, archéologue, en sont les principaux organisateurs et rédacteurs : trois et onze articles respectivement. Ils ont su s'entourer de personnes très au fait des domaines qu'ils traitent et ceci donne richesse et vie à l'ensemble. Si la longueur et l'écriture des articles diffèrent, l'unité est donnée par un même souci de faire entrer pleinement le lecteur dans chacun des thèmes abordés. À tous, novice ou amateur éclairé, est offert ainsi la possiblilité d'aborder, avec sûreté et plaisir, des rivages lointains qu'il est rarement donné d'approcher.

L'avant-propos expose le contexte dans lequel les collections uvéennes se sont constituées. D'entrée de matière, l'ouvrage met en lumière la particularité de l'étude des collections océaniennes. À la rareté des objets collectés par des missionnaires — pour la plupart ici, des militaires et quelques administrateurs, dont le cas exceptionnel de L.-J. Bouge — s'ajoute, pour la Polynésie occidentale notamment, la difficulté à déterminer avec certitude la provenance des pièces. Wallis, souvent associée à Futuna, illustre la nécessaire prise de conscience de la circulation d'éléments culturels au sein de ce « parfait ensemble de zone d'échanges culturels intenses ». Les îles n'ont cessé de s'influencer depuis toujours, rappelle-t-on, d'où le besoin d'assouplir certaines classifications régionales. Ici sont habituellement définis trois ensembles culturels : Fidji, les îles Samoa (qui surtout influencèrent Futuna) et les Tonga (ayant pour leur part marqué 'Uvea, autrement dit Wallis).

De ces premières remarques découle la logique de l'organisation de l'exposition, et du catalogue, en trois parties. Le visiteur, et le lecteur, sont en premier point instruits du maillage de Terre et d'Histoire auquel l'île appartient, comme le rappel Marie-Claire BatailleBenguigui. Cette spécialiste de la culture polynésienne aborde le passé de la zone avant que Daniel Frimigacci et Frédéric Angleviel, chacun dans leurs domaines, nous introduisent à l'histoire et la culture même de l'île. C'est ainsi que nous est retracé ce que fut l'aventure humaine du peuplement, pour les pécheurscéramistes Lapita, au cœur de ce "vaste et premier complexe micro-insulaire polynésien ». Frédéric Dupuy et Hélène Guiot donnent les nécessaires repères de géographie humaine. Le tout introduit au descriptif des pièces en provenance d'îles alentour: Futuna, Tonga, Samoa, Fidji, Tokelau, Niue, Rotuma.

Sur les $96 \mathrm{~km}^{2}$ de terres volcaniques qui la constitue, 'Uvéa l'ancienne, rebaptisée Wallis, dit l'histoire du travail des hommes ; c'est le thème de la seconde partie. Cette odyssée humaine a pour commencement l'implantation d'une population céramiste, notamment à 'Utuleve. Ce site du district de Mu'a donnera son nom à trois séquences à céramique de la préhistoire uvéenne jalonnée de périodes successives, étudiées et présentées par l'archéologue Daniel Frimigacci. Celle-ci débute, entre 1370 et 1320 avant J.-C., par un habitat, peut-être sur pilotis, qui se situe face à des passes du récif, sur des plages de galets et dunes, à proximité de zones marécageuses. À ces périodes où se mettent en place, à partir d'une « culture ancestrale : Utuleve I », des « différenciations culturelles régionales marquées par une diversification des types céramiques et la perte des décors Lapita : Utuleve II », succède une étape "dite de formation des systèmes de chefferie à titre : Utuleve II ». Aux alentours de l'an mille de notre ère, apparaît dans les marges d'un empire tongien en formation la "période d'Atulavu » : nom d'un site contemporain du règne du chef (hau) mythique Puhi. À celle-ci succède la « période des forts », vers 1400, « avec l'apparition de structures fortifiées tongiennes », l'abandon de la céramique et 
une abondante tradition orale. Enfin, en 1460/1500, s'installe la dynastie des Takumasiva sur laquelle s'appuient toujours la plupart des structures sociales actuelles, ainsi que plusieurs articles de la dernière partie le soulignent.

Autre particularité de ce territoire : la permanence ; elle ressort, malgré les bouleversements provoqués par l'arrivée des Occidentaux. Ses étapes furent ressenties très différemment à travers le Pacifique, selon l'importance des lieux et leur position par rapport aux nouvelles voies d'échanges se mettant en place qui négligaient totalement l'archipel. Cette période des premiers contacts (1767-1867) est ici celle de l'évangélisation. Les répercussions inévitables sont dépeintes, de façon claire et détaillée, par C. Stéfani et l'historien F. Angleviel, pour la culture matérielle notamment. H. Guiot complète ces pages par un rappel des principaux repères historiques entre les années 1887 et la fin des années 2000. Les mutations sont illustrées d'objets quasi emblématiques pour lesquels le catalogue apporte quelques précisions: lames d'herminettes et herminette, maquettes de pirogues, massue ou appuie-nuque royal, tapa et cachet royal, corbeille ou chasse-mouches présentés en parallèle avec une documentation qui trahit l'approche européenne de ces terres : exploration, évangélisation, éducation, législation.

La troisième partie, la plus importante, est consacrée à la société et sa culture, particulièrement à travers des productions $\mathrm{du} \mathrm{XIX}^{\mathrm{e}}$ siècle. Elle débute par ces piliers, au sens propre et figuré, que sont pour la société : la maison et la chefferie, pour reprendre l'idée habilement commentée par H. Guiot. Leur succède la langue (C. Moyse-Faurie) et le kava : vie du roi, vie de la société (S. Chave). Tous deux, à leur façon, alimentèrent la circulation des idées au sein de la tradition. $\mathrm{La}$ continuité culturelle est soulignée par l'approche faite de la religion et de la guerre (à travers les jeux) par H.Guiot. C'est ensuite un enchaînement d'études très nourries qui se poursuit par une approche de l'homme dans ses modes d'expression et de protection dont il s'entoure. Les étoffes d'écorce (P. Cusenier) avec lesquelles il se pare tout autant qu'il le vêt, puis les parfums et couleurs de son univers intime (H. Guiot) : de la maison "monde dans le monde » (S. Chave) à celui qui l'environne (F. Dupuy, H. Guiot) et le nourrit (H. Guiot) ; c'est-à-dire du choix et sens de la charpente aux implantations des plantes cultivées dans les jardins itinérants. Enfin, puisque la mer se présente comme le chemin le plus sûr pour aller des hommes vers d'autres hommes, c'est par la présentation de techniques de pêches et fabrication de pirogues que se termine le catalogue. L'exposition, quant à elle, présentait, pour conclure, un documentaire sur Wallis réalisé par l'antenne RFo locale (Radio France OutreMer). Le lecteur du catalogue, pour sa part, pourra poursuivre sa lecture par le détail des notices de cette dernière partie riche des rares objets qu'il est possible de présenter à l'heure actuelle. Un lexique permet utilement de se remémorer les termes des descriptions vues en route et une bibliographie variée soutiendra le lecteur curieux, ou l'amateur éclairé, dans ses recherches ultérieures.
À la lecture de ce catalogue, on trouve autant de plaisir que d'intérêt.

Marie-Noëlle OtTINo-GaRANGER

Ethno-archéologue

SHINEBERg, Dorothy, 1999. The people trade : Pacific island laborers and New Caledonia, 1865-1930, Honolulu, University of Hawaii Press, 309 pages, illustrations, cartes, tableaux, bibliographie, index.

Nos lecteurs n'ont pas oublié le livre pionnier de D. Shineberg, They came for sandal wood (Melbourne 1967, traduction française Nouméa 1973), non plus que divers articles sur la Nouvelle-Calédonie parus dans notre journal. Cette fois, après plus de dix années de recherches, elle publie ce qui restera longtemps l'ouvrage de référence sur le recrutement de la maind'œuvre insulaire pour les entreprises et les familles calédoniennes. C'est probablement pour « ratisser large » que l'éditeur américain a imposé à l'auteur ce titre aussi vague que malencontreux. Car, ne nous y trompons pas, c'est d'ouvriers et de domestiques qu'il s'agit, et non pas de « gens » qui, comme les Kosovars aujourd'hui ou les Irlandais du siècle dernier, quittent leur pays à la recherche d'un Eldorado.

Pendant les soixante-cinq années ici considérées, ils ont été plus de 15000 à être embauchés à destination de Nouméa. A $90 \%$ ils venaient des NouvellesHébrides (Vanuatu), foyer de recrutement préféré des colons et de l'administration coloniale. Ils étaient connus pour leur docilité, leur zèle au travail et leur incapacité à résister collectivement aux brimades de leurs maîtres. Leur seul travers, à bien regarder, était une certaine tendance à l'alcoolisme, mais largement compensée par leur propension à dépenser sur place tout ce qu'ils pouvaient gagner. Ce qui nous vaut inévitablement les tirades des employeurs blancs hésitant avec une bouffonnerie sinistre entre la maind'œuvre pénale, les Kanaks locaux, les Javanais et les Indochinois, autant d'êtres humains passablement dangereux, mais hélas tous tellement plus chers ! C'est là que l'Hébridais indiscutablement faisait prime sur le marché aux yeux des connaisseurs.

Moyennant quoi, on acceptait de passer par «pertes et profits » tout à la fois un taux de mortalité effarant (une recrue sur quatre), les risques inhérents à la distribution massive de fusils modernes à des « sauvages » belliqueux et toutes les petites et grandes tromperies permettant d'échapper aux contrôles de la police ou de la marine de guerre, notamment britannique.

D. Shineberg retrace en détail tous ces comportements et les calculs sous-jacents en une quinzaine de chapitres regroupés en trois parties : $1^{\circ}$ le recrutement, $2^{\circ}$ portrait des recrues et $3^{\circ}$ vie sur le lieu de travail. Comme les autres historiens du Pacifique se sont surtout occupés de ces pratiques dans les colonies britanniques et ne les ont que très rarement étudiées dans les territoires français, à l'exception notable de Slavers in Paradise (1981) de H. Maude, le livre que voici comble 